Des essais de consommation donneront toutes ces indications. Il faudra peser la cage et les attaches, tous les wagonnets vides et les produits d'extraction, et mesurer la puissance par le moteur et les services auxiliaires.

Les essais durent généralement une heure et commencent quand le moteur est en régime de température. La cage descendante devra être chargée de wagonnets vides. Les résultats d'essais s'expriment en kilowattheures par tonne hectométrique. Dans le cas du diagramme figure 4 , les consommations garanties sont de $0,586 \mathrm{kWh}$. par tonne hecto-métrique (temps d'enlevage 15 secondes). Il arrive de les exprimer en $\mathrm{kWh}$. par chevalheure. Pour passer d'un système à l'autre, il suffit de tenir compte du rapport ci-après :

$$
\frac{1.000 \times 100}{75 \times 3.600}=\frac{1}{2,7}
$$

La consommation en $\mathrm{kWh}$. par $\mathrm{CV}$.-heure utile à la cage est donc : 1,58 .

Ces chiffres montrent clairement que la machine d'extraction a vapeur ne peut nullement rivaliser avec la machine d'extraction èlectrique.

Les essais de consommation peuvent être effectués avec beaucoup de précision. Un compleur étalonné suffit. Un wattmètre enregistreur donne aussi les indications précises en ce qui concerne l'allure du trait. Nous avons dit antérieurement ce qui, dans l'installation de la machine, pouvait influer sur la consommation. Il convient d'ajouter que le rendement du puits est aussi un facteur important et il importe de ne pas le perdre de vue.

D. F. G.

Revue Jeumont (juillet-septembre 1929).

\title{
Méthode rapide, semi-expérimentale, pour la détermination du courant de cour-circuit permanent dans un réseau quelconque
}

\author{
par G. Crivalleri
}

Le développement de l'industrie électrique a provoqué l'établissement de lignes de transport très importantes à haute et très haute tension, qui relient des centres de production d'énergie électrique se trouvant à plusieurs dizaines de kilomètres l'une de l'autre, et permettent l'échange réciproque d'énergie suivant les besoins.

Les installations qui ont été reliées à ces grands réseaux ressentent infailliblement l'influence de ce réseau dans leurs caractéristiques propres de fonctionnement et particulièrement pour l'intensité du courant de court-circuit, dont la valeur dans la nouvelle condition a augmenté notablement.

Ce phénomène d' " accroissement " oblige les constructeurs à remplacer tous les appareils d'interruption préexistant, car souvent ils se sont démontrés insuffisants pour les nouvelles conditions de fonctionnement,

Même si ces appareils, qui doivent être remplacés, trouvent leur utilisation dans une autre installation, il n'en est pas moins ennuyeux d'être obligé de faire ce remaniement, surtout quand la place disponible est limitée et que cette transformation oblige à agrandir les bâtiments pour que les nouveaux appareils puissent y être installés. Les perturbations qui en résultent sont très fâcheuses et très coûteuses; et ces inconvénients ne sont pas moins graves au point de vue technique, car toutes ces modifications doivent être effectuées sans interrompre le service de la ligne.

Aussi sera-t-il utile d'éviter ces inconvénients en installant des interrupteurs proportionnés à la caractéristique de fonctionnement imposée par le réseau auquel l'installation en question doit se rattacher, et en prévoyant dans les limites logiques les futures augmentations possibles.

Les caractéristiques de l'interrupteur seront naturellement, suivant les considérations indiquées plus haut, établies par l'ingénieur qui fait le projet. L'indication du type d'interrupteur à choisir dans ces conditions peut être faite soit par l'ingénieur, soit par le constructeur qui fait l'offre; or les avis de ces deux techniciens ne concordent pas toujours; l'un se base sur les résultats qu'il déduit de ses calculs, l'autre sur une série de diagrammes établis soit par la pratique, soit par les essais faits sur des installations construites dans ce but. Ceci provoque assez souvent une discussion plus ou moins désintéressée, qui n'est pas toujours en faveur de celui qui finance l'entreprise.

Parmi les particularités d'un interrupteur, les plus usuelles: distance minimum dans l'air ou dans lhuile, nombre d'interruptions par phase, genre de contact, etc, il faut prendre en considération la pression intérieure à laquelle, dans les conditions les plus défavorables, l'interrupteur doit résister à la coupure d'un courant de court-circuit déterminé. Ceci mérite d'être noté, vu la tendance moderne d'augmenter le rapport entre la hauteur d'huile au-dessus des contacts et les dimensions transversales-de l'interrupteur, conditions pour lesquelles peut se produire le phénomène du piston d'huile. C'est une caractéristique que plusieurs constructeurs d'interrupteurs prennent en considération pour les types modernes à bac cylindrique de $300.000 \mathrm{kVA}$. ̀̀ $1.250 .000 \mathrm{kVA}$. et pour des tensions variables de 50 à $150 \mathrm{kV}$.

Cétte nouvelle caractéristique qui s'introduit depuis peu de temps, rend un bon nombre d'interrupteurs courants incapables d'assurer le service pour leçuel ils étaient prévus; si le constructeur garantit pour son interrupteur cette nouvelle caractéristique, la discussion sur le choix de l'interrupteur se resserre.

Ceci étant posé, abordons le sujet principal de l'étude : le courant de court-circuit permanent, celui que, généralement, l'interrupteur est appelé à couper. Le courant transitoire de court-circuit dans un réseau à haute tension est bien plus élevé que le courant permanent; mais on sait qu'il faut, en raison de la sélectivité, que tout interrupteur ait un retard de déclenchement pour permettre aux interrupteurs en aval de déclencher tout d'abord, ce qui- fait que, généralement, l'interrupteur en question n'entre en action que lorsque le régime transitoire est passé.

Pour les parties dérivées d'un grand réseau à moyenne tension et de quelques kilomètres de longueur, l'impédance de cette 
partie de ligne a une valeur nettement supérieure à la réactance synchrone du groupe générateur équivalent à tout le système des centrales en parallèle, ce qui fait que le courant de courtcircuit permanent est du même ordre de grandeur que le courant instantané. Le courant de court-circuit permanent ainsi calculé correspond au courant que l'interrupteur doit couper, que son déclenchement soit instantané ou retardé.

Ces considérations nous ont porté à prendre en considération uniquement le courant de court-circuit permanent.

Úne étude de la période transitoire du courant est utile pour pouvoir connaître l'effort électrodynamique auquel sont soumis les constructeurs et pour en déterminer la section minimum, ainsi que les contacts des interrupteurs afin d'éviter qu'ils atteignent des températures trop élevées dans l'intervalle entre la production du courtcircuit et le déclenchement des interrupteurs.

Pour la détermination du courant de courtcircuit permanent en un point quelconque du réseau, on peut se rapporter à plusieurs ouvrages éminents.

Tout en ne voulant pas tous les citer, ce qui nous serait d'ailleurs très difficile et ne voulant aucunement diminuer la valeur de ceux que nous omettrons, uniquement parce que nous n'avons pas eu l'occasion de les consulter, nous nous bornerons à citer les suivants : "Le correnti di corto circuito vei grandi impianti " et "Le sovra correnti nel macchinario elettrico ", de l'ingénieur Agostino Dalla Verde; "Directives pour le choix des interrupteurs des installations à courant alternatif à haute tension " de l'Association Suisse des Electriciens. Le traité classique de Biermanns. La monographie de Rüdemberg. Les articles de Charpentier qui comportent plusieurs exemples numériques et qui facilitent beaucoup la compréhension de la méthode analytique du calcul du courant de court-circuit. Très intéressant aussi est le traité de Fallou sur la méthode de coordonnées symétriques.

Tout en ayant sûrement omis d'autres traités tout aussi importants, on voit que la bibliographie sur l'étude que nous entreprenons est assez vaste et il est d'autant plus regrettable de constater que ces notions systématiques ne sont que très rarement appliquées dans la pratique.

C'est précisément cette considération qui nous pousse à publier cette étude si intéressante, qu'on pourra partager suivant les différentes méthodes proposées par les traités précités, savoir :

Méthodes analytiques;

Méthodes par ligne artificielle;

Méthodes expérimentales.

Dans les méthodes analytiques, l'impédance des machines et des différentes branches du réseau sont exprimées en ohms, en les rapportant à une tension de base qui, généralement, est celle du point auquel on veut déterminer le courant du courtcircuit.

Dans un circuit simple dans lequel les différents éléments sont en série, le courant du court-circuit se détermine facilement. C'est le quotient entre la tension indiquée et la somme des impédances.

Il est beaucoup plus facile de contrôler l'exactitude des calculs si on exprime les impédanees en chutes de tension relatives $(\%)$ rapportée à une puissance de base $\mathrm{P}$, arbitrairement choísie et exprimée en KVA.

La puissance de court-circuit $P_{c} / c$ en KVA au point considéré est exprimée par :

$$
\mathrm{P}_{\mathrm{c} / \mathrm{c}}=\frac{100 \mathrm{P}}{m} \mathrm{KVA}
$$

où $m$ est la somme des chutes de tension relatives.

Il est évident que $m$ doit être une somme géométrique des vecteurs représentant les chutes de tension, étant donné que celles-ci ne sont pas en phase; mais en pratique on fait une

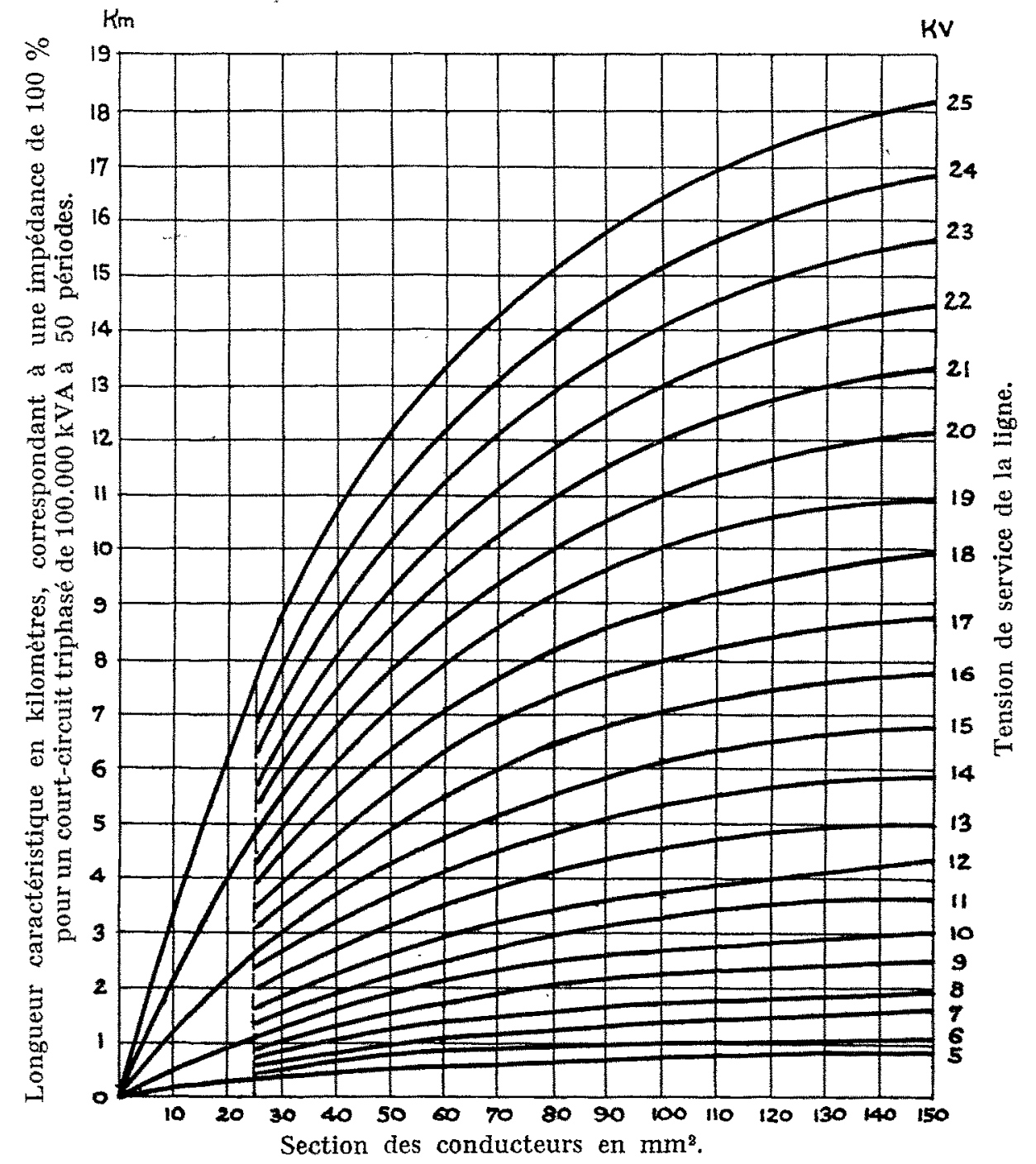

FIG. 1.

somme algébrique, ce qui fait commettre une erreur qui, pratiquement, n'est pas trop grande; la valeur du courant de courtcircuit obtenue est en effet légèrement inférieure à la valeur réelle.

TABleaU I

Distances entre les conducteurs en fonction de la tension du rëseau :

\begin{tabular}{|c|c|c|c|c|c|c|c|c|}
\hline Tension triphasée en KVA & 5 & 10 & 25 & 50 & 70 & 100 & 135 & 220 \\
\hline $\begin{array}{c}\text { Distance en mètres } \\
\text { entre les conducteurs }\end{array}$ & 0,5 & 0,75 & 1,00 & 1,25 & 1,50 & 3,00 & 5,00 & 7,50 \\
\hline
\end{tabular}

Dans le but de faciliter l'emploi de la méthode analytique, nous avons établi deux graphiques qui donnent rapidement la longueur en kilomètres que doit avoir une ligne triphasée 
pour qu'il y ait une chute de tension de $100 \%$ avec une puissance de court-circuit de $100.000 \mathrm{kVA}$., en fonction de la tension de fonctionnement et de la section des conductions (en cuivre) fig. 1 et 2 .

Soit une ligne a $50 \mathrm{kV}$. de $100 \mathrm{~cm}^{2}$ de section des conducteurs et de $120 \mathrm{~km}$ de longueur. Choisissons la puissance de base de la méthode analytiçue est très facile, et les résultats sont suffisamment justes. On peut s'en rendre compte en utilisant la méthode proposée par l'Association Suisse des Electriciens, c'est-à-dire en exprimant les impédances en ohms. De même, le résultat ne varie pas si on exprime les impédances en chutes de tension en pour cent. On arrive facilement au résultat cherché s'il s'agit d'une ligne simple : réseau du type radial. Quand le réseau est constitué par plusieurs centrales bouclées, le problème reste toujours déterminé; mais, pour le résoudre, il faut établir un système d'équations à plusieurs inconnues, dont la résolution, tout en n'etant pas chose très difficile, devient plutôt le travail d'un mathématicien que celui d'un ingénieur dont le but est d'arriver au résultat par le moyen le plus simple et le plus rapide, pourvu que celui-ci soit assez exact pour une application industrielle.

D'autre part, la recherche suivant la méthode classique et pédagogique donne un résultat d'une exactilude plutôt illusoire, car les impédances, paramìtres des equations qui constituent le système, doivent être exprimées en fonction des conditions les plus désavantageuses, en cas de court-circuit.

Pour pouvoir trouver effectivement les valeurs les plus désavantageuses des impédances, il faut refaire les calculs pour deux.ou trois situations différentes que l'intuition du technicien croit les plus intéressantes. Même cette précaution ne nous porte pas à des résultats sans critique, car si les impédances des transformateurs et de la ligne ne sont pas fonction de la charge, elles ne le sont pas pour les autres machines. Pour chacune d'elles, la réactance synchrone considérée n'est valable que pour des conditions d'excitation et de charge déterminées et non pas pour celles qui résultent de la répartition de la puissance du court-circuit dans la ligne, dans chaque machine.

$\mathrm{Si}$ on considère que dans les différents réseaux, on compte par dizaines le nombre des machines qui fonctionnent en parallèle et qui sont soumises à des conditions constamment variables, on voit combien il sera difficile d'arriver au résultat indiscutable, même si la réalisation du système établi est au point de vue mathématique sans reproches.

Nota. - Pour les lignes à $220 \mathrm{kV}$. avec conducteur en aluminium (environ $\Phi=25 \mathrm{~mm}$, distance entre conducteurs: $7 \mathrm{~m} 50$ ), la longueur caractéristique en kilomètres varie de 1.050 à 1.150 , suivant que les conducteurs sont sur un même plan ou forment un triangle.

$\mathrm{P}=20.000 \mathrm{kVA}$. L'impédance en pour cent rapportée à cette puissance nous est donnée par :

$$
\frac{120}{64} \times \frac{20.000}{100.000}=0,37=37 \%
$$

Dans le diagramme fig. 2, nous trouvons que c'est $64 \mathrm{~km}$., la longueur caractéristique pour ce genre de ligne.

Si le groupe des machines, générateur et transformateur, a respectivement une réactance synchrone de $40 \%$ et une tension de court-circuit de $10 \%$ la puissance de court-circuit du point considéré sera :

$$
\mathrm{P}_{\mathrm{c} / \mathrm{c}}=\frac{20.000}{0,40+0,10+0,37}=\frac{20.000}{0,87}=23.000 \mathrm{KVA}
$$

Avec l'emploi des courbes ci-jointes, on voit que l'application
Ce ne sont pas encore ces seules considérations qui peuvent nous induire en erreur, nous devons y ajouter d'autres, d'ordre encore plus général, celui dû à la méthode de détérmination de la réactance synchrone. Car la réactance synchrone qu'on connaît d'une machine est celle qu'on détermine à ses bornes où la tension est nulle. Mais dans le cas d'un court-circuit en un point quelconque de ligne, la tension aux bornes de la machine n'est pas nulle et l'impédance synchrone qui correspond à ces conditions n'est pas celle qu'on connaît. Une autre erreur par défaut dérive du fait qu'on rapporte les impédances pour une excitation et une charge déterminée à la tension en charge et non à la force électromotrice à vide. Car si cela n'apporte pas une erreur pour le calcul du courant de court-circuit aux bornes de la machine, l'erreur augmente proportionnellement à la 
distance qui sépare la machine du point où le court-circuit st produit.

Toutes ces erreurs inévitables ou presque et qui sont de même signe peuvent conduire, pour des cas spéciaux, à une valeur du courant de court-circuit inférieure même de $100 \%$ à celle réelle (voir Rüdemberg).

Cette considération est largement suffisante pour qu'un ingénieur se permette des licences, même en mathématiques.

Pour éviter aux calculs purement mathématiques et souvent assez compliqués dan le cas des réseaux bouclés, on se rapporte à la méthode de ligne artificielle, en reconstruisant le réseau en miniature et en remplaçant l'impédance de chaque générateur, transformateur et élément de ligne par une résistance proportionnelle. On alimente alors ce petit réseau par une batterie, en mettant un pôle à terre et connectant l'autre pôle aux points qui représentent les centrales. Le courant de court-circuit en un point du réseau sera représenté suivant une certaine proportionnalité par le courant qui se vérifie dans le réseau reproduit, lorsque le point correspondant de cette reproduction est mis à la terre.

Cette méthode permet d'étudier comment se répartit le courant de court-circuit dans les différentes centrales et dans quelles proportions, pour aboutir au point considéré. Staveren a perfectionné le système en remplaçant les simples résistances qui représentent les impédances du réseau par des groupes de résistances et réactances omises en série et proportionnées à celles qu'elles remplacent et en alimentant par du courant alternatif. Car l'hypothèse qu'on avait admise au début, c'est-à-dire que $m$ est la somme algébrique des chutes de tension dans le cas d'un réseau compliqué qui comporte des lignes aériennes et souterraines, donne souvent des résultats très différents de ceux qui correspondent à la réalité.

Tous les efforts faits pour créer et perfectionner cette méthode, qui, automatiquement, nous donne les résultats cherchés, démontrent la nécessité de détourner les techniciens des méthodes trop pédagogiques et peu pratiques. Une autre méthode encore plus directe est celle qui consiste à produire le court-circuit sur le réseau et à relever par des oscillographes le courant au point considéré. Mais cette méthode n'est pas très sympathique aux industriels, qui ne désirent guère ajouter aux incidents involontaires d'autres volontaires. Quand on applique cette méthode, on doit employer des relais perfectionnés qui, au moment du court-circuit, séparent la ligne en essai du reste du réseau.

Un essai de ce genre a été effectué sur la ligne de $110 \mathrm{kV}$. de la Bayernwerk A. G. de Münich pour contrôler le fonctionnement des relais sélectifs à distance Brown Boveri.

Ayant passé en revue les différentes méthodes de détermination du courant de court-circuit, il en résulte que les méthodes analytiques peuvent être toujours employées dans un calcul d'avant-projet d'une installation nouvelle qui n'est pas destinée à marcher en parallèle avec d'autres préexistantes.

Le cas qui, généralement, se présente est celui d'une installation nouvelle qui devra fonctionner avec d'autres installations déjà en exercice, d'où la nécessité de déterminer le courant de court-circuit qui pourra se produire dans ces conditions et auquel doit correspondre l'appareillage de l'installation nouvelle et celui de l'ancienne.

Dans ce cas, on utilisera la méthode semi-expérimentale qui nous donnera un résultat qui, tout en n'étant pas rigoureusement exact, est suffisant pour les besoins de l'industrie.

Le principe de calcul sur lequel se base la méthode semi-expérimentale est simple et dérive de la méthode analytique avec chute de tension en pour cent. En effet, supposons que tout calcul fait, on ait trouvé, pour la puissance de base de $50 \mathrm{kVA}$., en un point du réseau, une chute de tension de $40 \%$. La puissance de court-circuit en ce point sera environ de :

$$
\frac{50.000}{40} \times 100=125.000 \mathrm{KVA}
$$

Si on provoque en ce point en conditions normales de tension et de charge, une demande plus intense de courant de même phase que celle du court-circuit éventuel qui pourrait se produire, correspondant à $5.000 \mathrm{kVA}$. par exemple, il en résulte une chute de tension de

$$
\frac{5.000}{125.000} \times 100=4 \%
$$

Par un procédé inverse, la méthode semi-expérimentale produit une chute de tension de $4 \%$ avec $5.000 \mathrm{kVA}$. et on en déduit qu'en ce point le courant de court-circuit produirait une puissance correspondante de :

$$
\frac{5.000}{4} \times 100=125.000 \mathrm{KVA}
$$

En principe, toute la méthode se résume dans cet exemple explicatif. Elle nous porte à un résultat un peu erroné mais par excès, ce qui est toujours un avantage pour la plus grande sûreté de l'installation.

Les résultats seront d'autant plus précis que la phase du courant d'essai sera plus proche de la phase du courant de courtcircuit.

La réactance des machines se trouvant sur le réseau étant toujours de beaucoup supérieure à leur résistance ohmique, chose qui est vraie aussi pour la ligne, lorsqu'il s'agit de tension très élevée, il en résulte que le $\cos \varphi$ du courant de court-circuit n'est guère supérieur à 0,5 . Etant donné ces considérations, on peut employer un courant d'essai à $\cos \varphi=0$ sans commettre une erreur supérieure d̀ $15 \%$ en employant ainsi les générateurs synchrones des centrales et les moteurs synchrones des sous-stations;

Si on a donc une machine synchrone à sa disposition au point de la ligne qu'on prend en considération; $\Delta I$ et $\Delta P$ étant les variations de courant en quadrature et d'énergie réactive au moteur synchrone: $\Delta \mathrm{V}$ la variation de tension, $\mathrm{V}$ la tension primitive, on aura :

$$
\begin{gathered}
I_{c} / c=\Delta I \frac{Y^{\prime}}{\Delta V} \\
P_{c / c}=\Delta P \times \frac{V}{\Delta V}
\end{gathered}
$$

Comme on le voit, on n'a pas besoin de connaitre tous les éléments du réseau, ce qui simplifie beaucoup et accélère: le travail. Un autre avantage de la méthode semi-expérimentale, c'est de connaître les caractéristiques réelles de l'installation sans être obligé d'interrompre le service du réseau, car une variation temporaire de la tension de $\pm 5 \%$ peut être facilement appréciée, même par les voltmètres du tableau, et cette variation est tolérable pour le réseau.

La méthode est dite semi-expérimentale, car, en effet, elle n'est basée qu'en partie sur l'essai, car nous admettons que ce qui se vérifie pour une variation de tension relativement faible, se reproduit dans les mêmes proportions pour une variation de $100 \%$ done pour le court-circuit. Cette hypothèse est exacte pour les réactances des différents éléments du réseau, des transformateurs et pratiquement très approximative pour une partie 
de la réactance synchrone des générateurs, celle correspondant à la self-induction.

Mais l'hypothèse n'est plus exacte pour l'autre partie de la réactance, celle d'armature, pour laquelle la valeur ne varie pas proportionnellement à la charge, par suite de la saturation de l'induction.

Ce qui fait que la valeur de la réactance d'armature qu'on admet est celle correspondant à la perméabilité magnétique de l'inducteur en charge et tension normales et non à celle de l'état de saturation qui correspond à l'état produit par le courtcircuit.

L'erreur à laquelle nous induit cette inexactitude de la méthode est d'autant plus petite que la valeur de la réactance d'armature est faible par rapport à la somme des autres réactances dont les variations restent proportionnelles quelle que soit la condition de charge. L'erreur que nous venons d'analyser est négligeable quand le court-circuit se produit loin sur la ligne.

'Cette erreur est, au contraire, très appréciable lorsqu'il s'agit d'un court-circuit aux bornes de la machine. Pour cela, pour déterminer les conditions de court-circuit dans une centrale qui fonctionne en parallèle avec d'autres, il faut ajouter au résultat obtenu par la méthode semi-expérimentale le courant de court-circuit de toutes les machines synchrones de la centrale, en considérant leurs caractéristiques et condition de régime de court-circuit aux bornes.

On peut évaluer le courant de court-circuit pour les machines de 1,8 à 3 fois le courant normal suivant que les machines sont rapides ou lentes.

Il est très important d'observer que si le courant en quadrature absorbé par les récepteurs est proportionnel à la tension appliquée, le courant de court-circuit qu'on détermine par la méthode semi-expérimentale se rapporte automatiquement à la force électromotrice du générateur et non pas à la tension aux bornes de celui-ci. En d'autres termes, cette méthode nous permet de déterminer le courant de court-circuit correspondant au rapport entre la force électromotrice du générateur et la réactance totale du système, sans que l'on soit obligé de connaître cette force électromotrice.

Quand le réseau qu'on étudie est très compliqué, on peut utiliser la méthode semi-expérimentale en remplaçant le système des différentes centrales par une centrale unique de puissance égale à la somme des puissances existantes. De même on remplace par un centre récepteur unique les différents récepteurs du réseau en un point tel, par rapport au point producteur, qu'il puisse provoquer la chute de tension E-V qu'il y avait effectivement au moment de l'essai.

Ayant ainsi indiqué les différentes causes qui nous induisent en erreur, voici la méthode à suivre pour l'application de cette méthode.

Dans ce qui a été dit, nous avons supposé avoir une machine synchrone pour provoquer la variation de tension désirée; mais cette condition n'est pas toujours réalisable. On peut toujours produire une variation de tension en diminuant la charge et en tenant compte de la variation de tension qui s'ensuit par rapport à la valeur du courant réactif correspondant à la diminution de charge produite. Cette méthode n'est pas recommandable et on ne l'emploie que quand il est impossible d'imaginer une autre méthode, car ainsi on dérange les usagers.

Si dans une sous-station on a deux groupes distincts ne foncnant pas en parallèle, on modifie, par des artifices quelconques, leurs tensions et on fait un couplage momentané en parallèle. On aura une tension résultante $V_{0}$ et un courant d'échange $\Delta I$.
$\mathrm{Si}$ les fonctions de chaque groupe avant le couplage étaient $\mathrm{V}_{1}$ et $\mathrm{V}_{2}\left(\mathrm{~V}_{1}>\mathrm{V}_{2}\right)$; les courants de court-circuit de chaque groupe seront :

$$
\mathrm{l}_{1} \mathrm{c} / \mathrm{c}=\frac{\mathrm{V}_{0}}{\mathrm{~V}_{1}-\mathrm{V}_{0}} \Delta I \quad \mathrm{I}_{2} \mathrm{c} / \mathrm{c}=\frac{\mathrm{V}_{0}}{\mathrm{~V}_{0}-\mathrm{V}_{2}} \Delta \mathrm{I}
$$

et le courant maximum dans une dérivation du réseau sera exprimé par :

$$
I_{0} c / c=I_{1} c / c+I_{2} c / c=V_{0} \Delta I\left(\frac{1}{V_{1}-V_{0}}+\frac{1}{V_{0}-V_{2}}\right)
$$

Un autre moyen consiste à se servir d'une machine synchrone installée dans une centrale et reliée au réseau en observation par une ligne sans charge propre.

Quand on fait un essai, une particularité importante à observer est de faire la lecture des tensions simultanément au primaire et au secondaire, ainsi, on peut déterminer avec un seul essai le courant de court-circuit au primaire et au secondaire. La comparaison des deux courants ainsi déterminée donne facilement, par différence, l'impédance du groupe de transformation. Si ce résultat correspond avec bonne approximation à celui connu sous forme de tension de court-circuit, on a un contrôle automatique de l'essai effectué.

Il est toujours recommandé d'effectuer plusieurs mesures et d'en prendre la valeur moyenne tout en observant que les écarts entre une mesure et l'autre ne soient pas trop grands, cas pour lequel il faudrait trouver la raison de cette anomalie.

Si à la sous-station convergent plusieurs lignes d'alimentation, on peut déterminer rapidement les courants de courtcircuit dans différents cas, en diminuant le nombre d'alimentation ou en les couplant momentanément en parallèle. Cette possibilité nous permet un calcul très rapide du courant maximum de court-circuit, d'après lequel on peut s'ingénier pour exécuter la meilleure disposition qui nous permettra de limiter le courant de court-circuit à la valeur normale de la capacité de rupture des disjoncteurs. Nous étant occupé des réseaux à très haute tension, le cos. du courant de court-circuit, en ce cas, est très bas, ce qui nous autorise, nous l'avons déjà vu, à négliger la résistance ohmique par rapport à la réactance.

F Si on doit faire des essais sur des réseaux à tension moyenne égale ou inférieure à 15 à $20 \mathrm{~kW}$. avec une section de ligne (en cuivre) inférieure à 50 ou $60 \mathrm{~mm}^{2}$ et en un point à quelques kilomètres de la sous-station, la résistance ohmique en ce cas ne peut pas être négligée. L'application de la méthode décrite devient plus compliquée.

Il faut, en effet, pour chaque essai, produire deux variations de tension $\Delta V_{1}$ et $\Delta V_{2}$ avec les variations correspondantes de charge $\Delta I_{1}$ et $\Delta I_{2}$ et déphasages respectifs $\cos \varphi_{1}$ et $\cos \varphi_{2}$; tout en cherchant que ces deux déphasages soient autant que possible différents l'un de l'autre. En indiquant par $\mathrm{R}$ la résistance et par $\mathrm{X}$ la réactance équivalentes à tout le système, on aura approximativement :

d'où

$$
\begin{aligned}
& \frac{\Delta V_{1}}{\Delta I_{1}}=R \cos \varphi_{1}+X \sin l_{\varphi_{1}} \\
& \frac{\Delta V_{2}}{\Delta I_{2}}=R \cos p_{2}+X \sin \varphi_{2}
\end{aligned}
$$

et

$$
R=\frac{\frac{\Delta V_{1}}{\Delta I_{1}} \sin \varphi_{2}-\frac{\Delta V_{2}}{\Delta I_{2}} \sin \varphi_{1}}{\sin \left(\varphi_{2}-\varphi_{1}\right)}
$$

$$
X=\frac{\frac{\Delta V_{1}}{\Delta I_{1}} \cos \varphi_{2}-\frac{\Delta V_{2}}{\Delta I_{2}} \cos \varphi_{1}}{\sin \left(\varphi_{1}-\varphi_{2}\right)}
$$


En faisant un essai avec cos. $\varphi=1$ et un autre avec cos. $\varphi_{2}=0$ on peut simplifier et on obtient :

$$
\mathrm{R}=\frac{\Delta \mathrm{V}_{1}}{\Delta \mathrm{I}_{1}} \quad \mathrm{X}=\frac{\Delta \mathrm{V}_{2}}{\Delta \mathrm{I}_{2}}
$$

Ayant ainsi déterminé les valeurs de $\mathrm{R}$ et de $\mathrm{X}_{1}$ la valeur du courant de court-circuit sera exprimée par :

$$
\mathrm{I}_{\mathrm{c}} / \mathrm{c}=\frac{\mathrm{V}}{\sqrt{\left(\frac{\Delta \mathrm{V}_{1}}{\Delta \mathrm{I}_{1}}\right)^{2}+\left(\frac{\Delta \mathrm{V}_{2}}{\Delta \mathrm{I}_{2}}\right)^{2}}}=\frac{\mathrm{V}}{V \overline{\mathrm{R}^{2}+\mathrm{X}^{2}}}
$$

Au lieu de l'ètre, comme on avait supposé jusqu'à ce moment, par :

$$
I_{c} / c=\frac{V}{\left(\frac{\Delta V_{2}}{\Delta I_{2}}\right)}=\frac{V}{X}
$$

Comme il n'est pas toujours possible d'exécuter deux essais avec des $\cos \varphi$ différents, d'autre part ne pouvant pas négliger la résistance ohmique de la ligne, on peut utiliser la méthode semi-expérimentale jusqu'aux formes secondaires de la sousstation d'alimentation et la méthode analytique des bornes au point considéré.

Si le réseau aval de la sous-station est à lignes rayonnantes, en désignant par I $c / c$ le courant de court-circuit déterminé par la méthode semi-expérimentale aux bornes du secondaire, on peut calculer la réactance triphasée équivalente à la partie amont du réseau par

$$
\mathrm{X}=\frac{\mathrm{V}}{\mathrm{I} / \mathrm{c}}
$$

Or, si $r$ et $x$ sont respectivement la résistance et la réactance triphasées de la ligne de la sous-station au point considéré, le courant de court-circuit en ce point sera très approximativement :

$$
\mathrm{I}_{0} \mathrm{c} / \mathrm{c}=\frac{\mathrm{V}}{\sqrt{r^{2}+(\mathrm{X}+x)^{2}}}
$$

Pour illustrer d'une façon plus exacte ce qu'on vient de dire, prenons un exemple numérique : soit une sous-station de transformation d'une puissance de $15.000 \mathrm{kVA}$. avec un rapport de transformation $50 / 12 \mathrm{kV}$. Sur le côté basse tension, se trouve un moteur synchrone de $5.000 \mathrm{kVA}$. On se propose de déterminer le courant de court-circuit en trois points différents : immédiatement avant et après la transformation et à $5 \mathrm{~km}$. de la sous-station sur une des lignes dérivées à $12 \mathrm{kV}$. La ligne est en cuivre de $3 \times 50 \mathrm{~mm}^{2}$, avec distance entre conducteurs de $75 \mathrm{~cm}$.

Si par la méthode semi-expérimentale, avec une charge du moteur synchrone de $200 \mathrm{amp}$., correspondant à $4.150 \mathrm{kVA}$., on a obtenu une variation de tension de $5 \%$ au secondaire et de $3 \%$ au primaire, on en déduit :

$$
\begin{aligned}
& \mathrm{P}_{\mathrm{Ic} / \mathrm{c}}=\frac{4.150}{\frac{\mathrm{u}}{3}} \times 100=138.333 \mathrm{KVA} \\
& \mathrm{P}_{\mathrm{II} \mathrm{c} / \mathrm{c}}=\frac{4.150}{5} \times 100=83.000 \mathrm{KVA}
\end{aligned}
$$

En rapportant ces puissances de court-circuit à la puissance normale du groupe de transformation :

$$
\begin{gathered}
X_{I}=\frac{15.000}{138.333} \times 100=10,8 \% \\
X_{I I}=\frac{15.000}{83.000} \times 100=18 \%
\end{gathered}
$$

D'après ce qui a été dit, la réactance du transformateur est

$$
\mathrm{X}_{\mathrm{T}}=18-10,8=7,2 \%
$$

et elle doit correspondre en valeur, si l'essai a été bien effectué à la tension de court-circuit en pour cent.

Pour calculer le courant de court-circuit au point distant de $5 \mathrm{~km}$. de la sous-station, on peut procéder de deux façons différentes :

Supposer l'impédance totale du réseau en amont du point considèré comme la somme arithmétique de l'impédance en amont de la sous-station et de l'impédance entre la sous-station et de l'impédance entre la sous-station et le point considéré; ou déterminer les valeurs de $\mathrm{X}, r$ et $x$, comme il a été indiqué plus haut et appliquer la formule connue.

Employons les deux méthodes :

a) Méthode avec les courbes des figures 1 et 2. - La longueur caractéristique de la ligne est de $2,850 \mathrm{~km}$. Pour la puisssance de base de $100.000 \mathrm{kVA}$, on aura les impédances en pour cent

$\mathrm{Z} \%$ en amont au secondaire $=\frac{100.000}{83.000} \times 100=121 \%$

$$
\mathrm{z} \% \text { de la ligne secondaire }=\quad \frac{5 \mathrm{Km}}{2,85} \times 100=175 \%
$$

$\mathrm{Z} \%$ de la ligne en amont du point

La puissance de court-circuit au point considéré est :

$$
\begin{gathered}
\mathrm{P}_{\mathrm{c} / \mathrm{c}}=\frac{100.000}{296} \times 100=33.800 \mathrm{KVA} \\
\mathrm{I}_{\mathrm{c} / \mathrm{c}}=\frac{33.800}{12.000 \cdot V^{\overline{3}}}=1.630 \mathrm{~A} .
\end{gathered}
$$

(A suivre)

L'Electrotechnica, 25 mars 1930. 